\title{
Is total elbow arthroplasty a reliable alternative treatment option for comminuted distal humerus fractures in elderly patients?
}

\author{
Myung-Sun Kim \\ Department of Orthopedic Surgery, Chonnam National University Hospital, Chonnam National University Medical School, Gwangju, Korea
}

The treatment of comminuted intra-articular distal humerus fractures is challenging in elderly patients. Open reduction and internal fixation (ORIF) in elderly patients has a high risk of complications, including poor functional outcomes, persistent pain, infection, stiffness, nonunion, ulnar neuropathy, internal fixation failure, and heterotopic ossification [1-3]. Because of poorer ORIF outcomes in osteoporotic elderly patients, there has been increasing interest in total elbow arthroplasty (TEA) as a more reliable alternative to ORIF and nonsurgical treatment.

Primary TEA for acute distal humerus fractures was first reported in 1997 by Cobb and Morrey [4]. They retrospectively reviewed the records of 20 patients ( 21 elbows) who had a mean age of 72 years at the time of injury. The mean duration of follow-up was 3.3 years (range, 3-10.5 years). Based on the Mayo elbow performance Score (MEPS), 15 elbows had an excellent result and five had a good result; there were inadequate data for one elbow. There were no fair or poor results. Several other studies have confirmed similar, consistently reliable results [5-7]. Prasad et al. [8] found that survivorship, with revision and definite loosening as end-points, was $89.5 \%$ at 10 years with male patients having a higher incidence of loosening and wear.

Frankle et al. [9] performed a retrospective comparison be- tween women older than 65 who underwent ORIF (12 patients) and TEA (12 patients) for distal intra-articular humerus fractures. All fractures were Orthopedic Trauma Association (OTA) classification 13.C2 or 13.C3. After a minimum of 2 years of follow-up, there were one good and 11 excellent results based on MEPS among those treated with TEA. There were no fair or poor outcomes. On the other hand, there were four excellent, four good, one fair, and three poor results (cases that required conversion to TEA) among those treated with ORIF. McKee et al. [3] conducted a prospective, randomized, controlled trial to compare clinical outcomes in elderly patients (older than 65 years, OTA classification 13C) with displaced intra-articular, distal humerus fractures treated with ORIF (15 patients, mean age of 77 years) or primary semi-constrained TEA (25 patients, mean age of 78 years). The TEA group resulted had more predictable and improved 2-year functional outcomes than the ORIF group. The fact that $25 \%$ of fractures randomized to ORIF were not amenable to internal fixation suggests that TEA may result in decreased reoperation rates. The authors ultimately concluded that TEA is preferred over ORIF in elderly patients with complex distal humerus fractures that are not amenable to stable fixation. Dehghan et al. [10] evaluated long-term outcomes (mean follow-up period 
of 12.5 years) of TEA for distal humerus fractures from a prior randomized clinical trial [3]. They concluded that TEA is an effective and reliable procedure for treatment of comminuted distal humerus fractures in elderly patients and a well-performed TEA will give them a well-functioning elbow for life. A systematic review and meta-analysis by Githens et al. [11] revealed that TEA and ORIF for the treatment of geriatric distal humerus fractures produced similar functional outcome scores and range of motion. Although there was a trend toward a higher rate of major complications and reoperation after ORIF, this was not statistically significant.

The paper titled "Comparison of open reduction and internal fixation with total elbow arthroplasty for intra-articular distal humeral fractures in older age: a retrospective study" by Lee et al. [12] in this issue of Clinics in Shoulder and Elbow compared the clinical and functional outcomes of ORIF (28 patients) and TEA (43 patients) in patients aged $\geq 65$ years. At the last follow-up visit (ORIF group: mean, 31 months; TEA group: mean, 34 months), 93\% (26/28 cases) of the ORIF group showed good-to-excellent results based on MEPS. On the other hand, only 35\% (15/43 cases) of the TEA group showed good results. This result is interesting because it is contrary to previous reports in the literature that TEA has better or similar clinical results compared to ORIF for complex distal humeral fractures.

Throckmorton et al. [13] reported the specific failure patterns after linked semi-constrained TEA for posttraumatic arthritis. They demonstrated a 15 -year survival rate of $70 \%$ with revision or resection for any reason as the end point with $68 \%$ good-to-excellent clinical results. The most common cause of early failure (failure after less than 5 years) was infection, whereas intermediate-term failure (failure after 5 to 10 years) typically was due to bushing wear. Late failure (failure after more than 10 years) was uncommon and involved component loosening or fracture. Seventy-five percent of failures occurred in patients younger than 60 years who had greater physical demands.

With regard to postoperative daily activities, TEA comes with a lifetime repetitive weight-lifting restriction of approximately 5 lb. In addition, heavy manual work, and forceful pushing and pulling activities are usually restricted. Therefore, TEA should be reserved for selected lower-demand elderly patients only; it is not an option for younger, higher-demand individuals. As the number of TEA procedures continues to increase, the revision burden will correspondingly increase [14]. The treatment of distal humerus fractures with osteoporotic and highly comminuted articular surfaces is often challenging. ORIF is the gold standard in younger patients and also should be the first-line treatment in elderly patients if osteosynthesis is possible. Therefore, TEA for the treatment of distal humerus fractures is indicated in elderly, low-demand patients and those with osteoporosis, pre-existing inflammatory arthritis, osteoarthritis, or a reduced life expectancy. It is also indicated for comminuted and nonrepairable articular surfaces, pathologic fractures, and fracture nonunion. The indications for TEA have expanded substantially in the past 10 years. Currently, acute trauma and posttraumatic arthritis of the elbow are the main indications for TEA, surpassing rheumatoid arthritis [15]. Although there are good long-term data regarding these prosthetic devices for rheumatoid arthritis, additional evidence is needed for traumatic situations because they pose a unique set of challenges and complications. Clinical evidence from long-term prospective randomized controlled studies is required to determine the feasibility of TEA in the treatment of complex distal humerus fractures in elderly patients.

\section{REFERENCES}

1. Jupiter JB, Neff U, Holzach P, Allgöwer M. Intercondylar fractures of the humerus: an operative approach. J Bone Joint Surg Am 1985;67:226-39.

2. Kundel K, Braun W, Wieberneit J, Rüter A. Intraarticular distal humerus fractures: factors affecting functional outcome. Clin Orthop Relat Res 1996;200-8.

3. McKee MD, Veillette CJ, Hall JA, et al. A multicenter, prospective, randomized, controlled trial of open reduction: internal fixation versus total elbow arthroplasty for displaced intra-articular distal humeral fractures in elderly patients. J Shoulder Elbow Surg 2009;18:3-12.

4. Cobb TK, Morrey BF. Total elbow arthroplasty as primary treatment for distal humeral fractures in elderly patients. J Bone Joint Surg Am 1997;79:826-32.

5. Garcia JA, Mykula R, Stanley D. Complex fractures of the distal humerus in the elderly: the role of total elbow replacement as primary treatment. J Bone Joint Surg Br 2002;84:812-6.

6. Lee KT, Lai CH, Singh S. Results of total elbow arthroplasty in the treatment of distal humerus fractures in elderly Asian patients. J Trauma 2006;61:889-92.

7. Giannicola G, Scacchi M, Polimanti D, Cinotti G. Discovery elbow system: 2-to 5-year results in distal humerus fractures and posttraumatic conditions: a prospective study on 24 patients. J Hand Surg Am 2014;39:1746-56.

8. Prasad N, Ali A, Stanley D. Total elbow arthroplasty for non-rheumatoid patients with a fracture of the distal humerus: a minimum ten-year follow-up. Bone Joint J 2016;98:381-6.

9. Frankle MA, Herscovici D Jr, DiPasquale TG, Vasey MB, Sanders RW. A comparison of open reduction and internal fixation 
and primary total elbow arthroplasty in the treatment of intraarticular distal humerus fractures in women older than age 65. J Orthop Trauma 2003;17:473-80.

10. Dehghan N, Furey M, Schemitsch L, et al. Long-term outcomes of total elbow arthroplasty for distal humeral fracture: results from a prior randomized clinical trial. J Shoulder Elbow Surg 2019;28:2198-204.

11. Githens M, Yao J, Sox AH, Bishop J. Open reduction and internal fixation versus total elbow arthroplasty for the treatment of geriatric distal humerus fractures: a systematic review and meta-analysis. J Orthop Trauma 2014;28:481-8.

12. Lee SH, Baik JS, Kang HT, Seong TH, Kim JW. Comparison of open reduction and internal fixation with total elbow arthroplasty for intra-articular distal humeral fractures in older age: a retrospective study. Clin Shoulder Elbow 2020;23:94-99.

13. Throckmorton T, Zarkadas P, Sanchez-Sotelo J, Morrey B. Failure patterns after linked semiconstrained total elbow arthroplasty for posttraumatic arthritis. J Bone Joint Surg Am 2010; 92:1432-41.

14. Cheung E, Nathani A, Tashjian R, Armstrong A, Morrey M. Elbow trauma sequelae: instability, stiffness, non-arthroplasty, and arthroplasty options. Instr Course Lect 2019;68:117-40.

15. Lapner M, King GJ. Elbow arthroplasty for distal humeral fractures. Instr Course Lect 2014;63:15-26. 